\title{
Movimentos de adaptação das atividades do Programa de Educação pelo Trabalho para a Saúde no contexto da pandemia de covid-19
}

\author{
Movements of adaptation of the activities of the Education through Work for Health Program in
}

the context of the covid-19 pandemic

Movimientos de adecuación de las actividades del Programa Educación por el Trabajo para la Salud en el contexto de la pandemia covid-19

Recebido: 12/05/2021 | Revisado: 18/05/2021 | Aceito: 19/05/2021 | Publicado: 06/06/2021

\author{
Rachel Brinco de Souza \\ ORCID: https://orcid.org/0000-0001-8563-3525 \\ Universidade do Estado do Rio de Janeiro, Brasil \\ E-mail: kelbrinco@yahoo.com.br \\ Carinne Magnago \\ ORCID: https://orcid.org/0000-0001-8799-3225 \\ Universidade de São Paulo, Brasil \\ E-mail: carinne@usp.br \\ Tania França \\ ORCID: https://orcid.org/0000-0002-8209-9811 \\ Universidade do Estado do Rio de Janeiro, Brasil \\ E-mail: taniafranca29@gmail.com
}

\begin{abstract}
Resumo
O Programa de Educação pelo Trabalho para a Saúde (PET-Saúde) visa reorientar a formação profissional em saúde a partir da inserção de estudantes nos serviços de saúde. Com a pandemia de covid-19 as atividades dos grupos tutoriais precisaram ser repensadas em função do interrompimento das atividades presencias. Este estudo de caso objetivou conhecer o processo de adaptação dos grupos tutorias para o desenvolvimento de atividades no contexto da pandemia de covid-19. Foram entrevistados 32 integrantes de projetos PET-Saúde do estado do Rio de Janeiro. Seis movimentos de adaptação foram identificados: replanejamento das ações, adaptação para o uso das tecnologias digitais, intensificação das atividades intragrupos, constituição de grupos de apoio, apoio às secretarias de saúde, e educação em saúde por meio das mídias sociais. Os movimentos de adaptação são flexíveis a ajustamentos, permitindo que os grupos reflitam e incorporem a complexidade do contexto pandêmico e as necessidades da comunidade no desenvolvimento das ações. As ações desempenhadas, planejadas e estruturadas coletivamente, respeitando-se as limitações e vulnerabilidades dos estudantes, preceptores e tutores, demonstram que é possível apoiar o enfrentamento da pandemia de covid-19, e ensinar e aprender a partir da realidade cotidiana dos serviços de saúde, mesmo não estando na linha de frente.
\end{abstract}

Palavras-chave: Serviços de integração docente-assistencial; Infecções por coronavírus; Pandemias; Educação interprofissional; Educação superior.

\begin{abstract}
The Education through Work for Health Program (PET-Saúde) aims to reorient professional training in health based on the inclusion of students in health services. The activities of the tutorial groups needed to be rethought due to the interruption of classroom activities because the covid-19 pandemic. This case study aimed to learn about the adaptation process of the tutorial groups for the development of activities in the context of the covid-19 pandemic. 32 members of PET-Saúde projects in the state of Rio de Janeiro were interviewed. Six adaptation movements were identified: redesigning actions, adapting to the use of digital technologies, intensifying intra-group activities, setting up support groups, supporting health departments, and health education through social media. Adaptation movements are flexible to adjustments, allowing groups to reflect and incorporate the complexity of the pandemic context and the needs of the community in the development of actions. The actions performed, planned, and structured collectively, respecting the limitations and vulnerabilities of students, preceptors, and tutors, demonstrate that it is possible to support the coping covid-19 pandemic, and to teach and learn from the everyday reality of health services, even if not in the front line.
\end{abstract}

Keywords: Teaching care integration services; Coronavirus infections; Pandemics; Interprofessional education; Education, Higher. 


\section{Resumen}

El Programa Educación por el Trabajo para la Salud (PET-Saúde) objetiva reorientar la formación profesional en salud a partir de la inclusión de los estudiantes en los servicios de salud. Con la pandemia del covid-19, las actividades de los grupos tutoriales debieron repensarse debido a la interrupción de las actividades presenciales. Este estudio buscó conocer el proceso de adaptación de los grupos de tutoría para el desarrollo de actividades en el contexto de la pandemia. Se entrevistó 32 integrantes de PET-Saúde en el estado de Río de Janeiro. Se identificaron seis movimientos: rediseño de acciones, adaptación al uso de tecnologías digitales, intensificación de actividades intragrupales, conformación de grupos de apoyo, apoyo a los departamentos de salud y educación para la salud a través de las redes sociales. Los movimientos son flexibles a los ajustes, permitiendo que los grupos reflejen e incorporen la complejidad del contexto pandémico y las necesidades de la comunidad en el desarrollo de acciones. Las acciones realizadas, planificadas y estructuradas colectivamente, respetando las limitaciones y vulnerabilidades de alumnos, preceptores y tutores, demuestran que es posible apoyar el afrontamiento de la pandemia, y enseñar y aprender a partir de la realidad cotidiana de los servicios de salud.

Palabras clave: Servicios de integración docente asistencial; Infecciones por coronavirus; Pandemias; Educación interprofesional; Educación superior.

\section{Introdução}

Nos mais de 30 anos do Sistema Único de Saúde (SUS) foram implementadas diferentes iniciativas de reorientação da formação de profissionais de saúde balizadas na educação permanente em saúde e nas Diretrizes Curriculares Nacionais para os cursos de graduação da área. Insere-se, nesse conjunto, o Programa de Educação pelo Trabalho para a Saúde (PET-Saúde), criado em 2008 com o objetivo de promover o desenvolvimento de atividades de integração ensino-serviço-comunidade. Para tanto, prevê a conformação de grupos tutoriais de aprendizagem, conformados por tutores (docentes), preceptores (profissionais do serviço) e estudantes da saúde, como instrumento de inserção e vivência desses últimos nos serviços do SUS (França, Magnago, Santos, Belisário, \& Silva, 2018).

Desde a sua criação, o programa teve nove edições lançadas, sendo a última delas o PETSaúde/Interprofissionalidade, configurada no sentido de promover a educação interprofissional (EIP), compreendida como um modelo pedagógico que propõe a aprendizagem compartilhada e interativa entre atores de diferentes cursos da saúde (França et al., 2018; Almeida, Teston, \& Medeiros, 2019). Por meio da EIP se aprende sobre os outros, com os outros e entre si visando ao desenvolvimento de habilidades e competências colaborativas que viabilizem o trabalho em equipe efetivo, resolutivo e alinhado às necessidades de saúde (Barr, 1998; Reeves, 2016).

Efetivamente iniciada em abril de 2019, com período de vigência previsto de 24 meses, a edição Interprofissionalidade aprovou 120 projetos propostos conjuntamente por Secretarias Municipais/Estaduais de Saúde e instituições de ensino superior, as quais deveriam desenvolver ações de integração entre os atores do SUS e a comunidade acadêmica, conforme delineado em seu projeto. Acredita-se, no entanto, que as atividades dos grupos tutoriais precisaram ser repensadas em função da pandemia de coronavirus disease (covid-19), que já matou quase 430 mil pessoas no Brasil (Conselho Nacional de Secretários de Saúde, 2021). Isso, pois, desde março de 2020, as instituições de ensino precisaram interromper as atividades presenciais em favor do isolamento social, uma das principais medidas adotadas para a contenção da doença (Garcia \& Duarte, 2020); e um ator basilar do programa, o preceptor, vem atuando na linha de frente da luta global contra a covid-19, potencialmente afetando o desenvolvimento das ações planejadas nos serviços de saúde.

Considerando a finalização da vigência da edição e a ausência de publicações sobre o programa no atual cenário de crise sanitária, este estudo objetivou conhecer o processo de adaptação de grupos tutorias do PET-Saúde/Interprofissionalidade para o desenvolvimento de atividades no contexto da pandemia de covid-19. 


\section{Metodologia}

Este estudo se configura como pesquisa exploratória e qualitativa, do tipo estudo de caso, desenvolvido no estado do Rio de Janeiro no período de maio a setembro de 2020. A população de interesse do estudo foi composta pelos integrantes de projetos PET-Saúde/Interprofissionalidade que tiveram como proponentes instituições de ensino também participantes da edição anterior do programa (PET-Saúde/GraduaSUS). Dos dez projetos do estado, cinco atendem a esse critério: o da Universidade Federal Fluminense (Niterói); dois da Universidade Federal do Rio de Janeiro (Macaé e Rio de Janeiro); um da Universidade do Estado do Rio de Janeiro (Rio de Janeiro); e um do Centro Universitário de Volta Redonda.

Os coordenadores de projeto foram convidados a participar da pesquisa e a eles solicitado que estendessem o convite a outros integrantes do PET-Saúde, sendo pelo menos dois estudantes de cursos diferentes, um preceptor e um tutor. Manifestaram desejo em participar os cinco coordenadores de projeto, 11 estudantes, nove preceptores e sete tutores que, juntos, conformaram a amostra multiprofissional do estudo $(n=32)$.

Os dados foram coletados por meio de entrevistas virtuais e individuais realizadas na plataforma de webconferência Zoom e gravadas com o consentimento de cada participante. A coleta dos dados foi orientada por um roteiro não estruturado, composto por questões relacionadas à condução das atividades dos grupos tutoriais no contexto pandêmico.

As entrevistas duraram, em média, 40 minutos, foram transcritas e submetidas às etapas usuais da análise de conteúdo (Bardin, 2011): pré-análise, fase em que o material foi organizado, lido e dele foram extraídas as principais ideias captadas; exploração do material, na qual os registros foram identificados, codificados e agrupados por similaridade; e, finalmente, inferência e interpretação das informações analisadas.

O projeto da pesquisa foi submetido e aprovado por cinco diferentes Comitês de Ética em Pesquisa, conforme resoluções de cada instituição participante, e obedeceu a todas as normas nacionais relativas a pesquisas envolvendo seres humanos.

\section{Resultados}

A partir das entrevistas foi possível constatar que os grupos tutoriais do estado do Rio de Janeiro trilharam e vêm trilhando um percurso similar desde o início da pandemia. Esse trajeto pode ser traduzido em seis movimentos simultâneos, interdependentes e flexíveis a ajustamentos, que permitem refletir e incorporar a complexidade do contexto pandêmico e as necessidades da comunidade no desenvolvimento das ações (Figura 1). 
Figura 1. Movimentos operados pelos grupos PET-Saúde/Interprofissionalidade do estado do Rio de Janeiro no contexto da pandemia. Rio de Janeiro, 2020

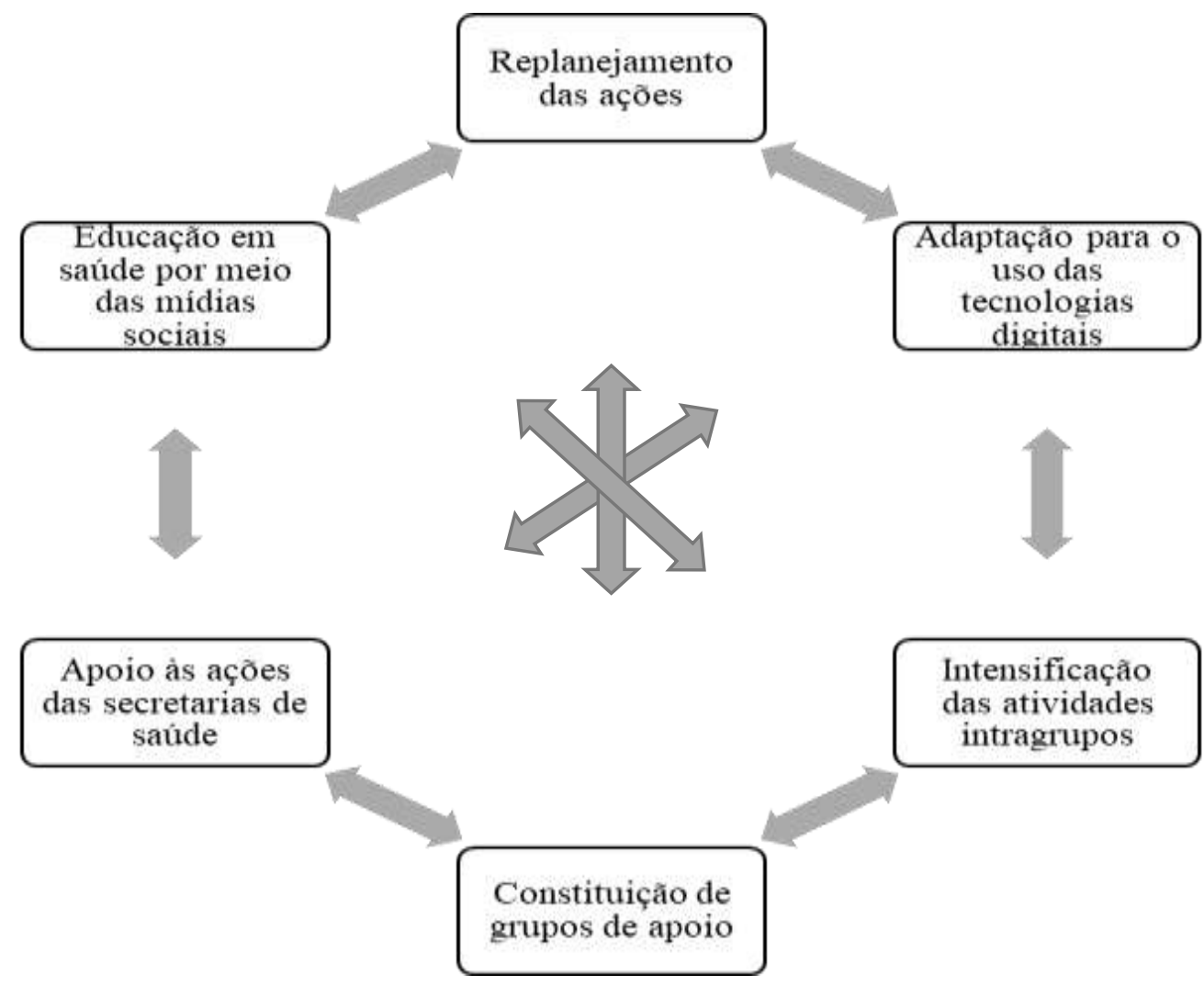

Fonte: Autores (2021).

Diante da situação de excepcionalidade provocada pela pandemia, os integrantes do PET-Saúde foram instados a empreender movimentos de replanejamento e de construção de estratégias não presenciais, que não apenas considerassem os objetivos do programa e os preceitos da EIP, mas também as demandas emergidas pela covid-19.

"A gente teve que se reorganizar nesse período, de forma muito abrupta, para que houvesse uma solução de continuidade do trabalho do PET-Saúde. Então, cada tutor ficou responsável por desenhar propostas com seus preceptores." (Tutor 2)

“Com a pandemia, tudo mudou por conta do isolamento social. A gente teve que fazer um outro recorte, dentro da perspectiva da interprofissionalidade, com os portadores da covid-19. Foi preciso reorganizar. " (Preceptor 3)

Se, por um lado, para algumas instituições de ensino e de saúde retirar os estudantes do território assistencial e remodelar as atividades presenciais para atividades virtuais e remotas foi tarefa basilar e consensual; para outras, houve dissenso sobre a atuação dos alunos nos serviços de saúde como fonte de apoio ao enfrentamento da pandemia, sobretudo em razão da escassez de profissionais para atendimento da demanda aumentada.

"Escrevi uma nota, enquanto assessor da secretaria municipal de saúde, que solicitava a retirada dos alunos das unidades, independentemente de ser PET-Saúde ou não, e organizava tarefas de monitoramento remoto de pacientes. [...] A gente apresentou a proposta para a prefeitura [...] e identificamos que algumas pessoas estavam reticentes, 
pois achavam que os alunos tinham que ficar lá para atender porque não tinha médico [...]. Mas, com o apoio da universidade, dos preceptores e tutores, ganhamos força e conseguimos virtualizar as atividades." (Coordenador de projeto 5)

Dado que o fio condutor do programa é a integração ensino-serviço-comunidade, o que, em primeira análise, imprime a realização de atividades nos cenários do SUS, a transposição das atividades do plano presencial para o virtual constituiu-se, de fato, um desafio. Desafio esse que exigiu, de maneira abrupta, movimentos de adaptação para o uso rotineiro de tecnologias digitais, e de compreensão dos problemas estruturais da vida real que limitam o acesso e a utilização dessas tecnologias pelos atores do PET-Saúde.

“Com a pandemia tivemos que, a toque de caixa, aprender a usar as tecnologias.” (Tutor 1)

"Fizemos um seminário via live, uma novidade. Todo mundo aprendendo a mexer com o YouTube [...]. A gente vai tentando se adaptar a esse novo cenário, com aula remota. É tudo muito novo e é assim que a gente tem feito, tudo online." (Preceptor 8)

"Nós temos estudantes de vários níveis socioeconômicos. Tem gente que está em casa otimamente bem, liga a internet e trabalha, mas tem gente que está cuidando do irmão, da avó, da tia." (Coordenador de projeto 3)

"Sempre tem um ou outro com alguma dificuldade de conexão, de falta de privacidade, pois não é só você ter inclusão digital, conexão e um computador. Temos que pensar nos que precisam estudar em uma casa de um quarto só, com vários membros da família." (Tutor 4)

Apesar dos percalços que envolvem o uso de tecnologias digitais, o trabalho remoto intensificou as atividades intragrupos e aumentou a frequência dos encontros, agora virtuais. Nesses momentos, os participantes do programa buscam planejar novas ações, estudar e aprender juntos, discutir casos e desenvolver pesquisas e produções bibliográficas.

“Todo mundo do PET-Saúde fez o curso do coronavírus disponibilizado pela Fiocruz e pelo Ministério da Saúde. A gente fez o curso e foi debatendo sobre o que aprendemos." (Tutor 6)

"Estamos escrevendo artigos, inclusive acabamos de submeter um sobre o NASF [Núcleo Ampliado de Saúde da Família] e a necropolítica em tempos de pandemia." (Acadêmico 8).

“Estamos tendo reuniões para a discussão de casos de pacientes dos CAPS [Centro de Atenção Psicossocial] que já estávamos acompanhando; e o que sai na discussão eu levo para a unidade, para os meus pacientes.” (Preceptor 6).

A amplificação dos encontros revelou aos integrantes do PET-Saúde como o contexto pandêmico e o isolamento social têm repercutido intensamente na sua vida coletiva e individual, desencadeando movimentos de ajuda mútua. Os encontros, agora virtuais, deixaram de ser apenas momentos formais de planejamento, de delineamento de ações e de ensino; passaram a ser grupos de apoio, abrindo espaço para o acolhimento e a escuta. 
"Estamos tentando fazer uma troca de vivências; de como tem sido para cada um passar por esse período de pandemia. É como se fosse um grupo de apoio, pois algumas pessoas estão afastadas da família, do trabalho, da faculdade. A gente leva uma poesia, fala sobre uma música." (Acadêmico 4)

"Estamos fazendo reunião virtual semanal para saber como cada pessoa está, porque a vida não está normal." (Acadêmico 6)

Nesse cenário, é preciso ressaltar o preceptor que, enquanto profissional de saúde, tem atuado incansavelmente na linha de frente, estando mais suscetível à infecção pelo novo coronavírus, assim como ao adoecimento mental, produto da sobrecarga de trabalho, estresse ocupacional e dificuldades em lidar com as adversidades dessa situação. Nesse contexto tem sido necessário ressignificar a sua dedicação às atividades do projeto.

"Estamos exigindo menos dos preceptores nesse momento, porque eu sei que muitos deles estão em um trabalho muito estressante." (Coordenador de projeto 3)

“Em um primeiro momento, os preceptores foram poupados de cuidar dos alunos. Não dava para pedir para eles organizarem atividades remotas com seus alunos, porque eles estavam loucos no meio da pandemia, muitos deles doentes." (Coordenador de projeto 5)

Ainda nessa perspectiva e cientes do impacto da pandemia na vida dos profissionais de saúde, esses movimentos de apoio extrapolaram os grupos tutoriais e se estenderam para a rede assistencial do território onde atuam.

“A gente tem um grupo de trabalho de saúde mental, por meio do qual a gente iniciou uma atividade de acolhimento de profissionais de saúde, pois eles estão passando por uma carga muito alta de estresse, de preocupação com a própria família e tudo o mais." (Acadêmico 7)

Mais ainda, os resultados demonstram que a pandemia desafiou os integrantes do PET-Saúde a propor e se engajar em estratégias que apoiassem as secretarias de saúde, contribuindo para melhorar o quadro sanitário imposto pela covid-19 e oportunizando o desenvolvimento de habilidades e competências profissionais requeridas pela situação sanitária.

“A gente participou de um telemonitoramento sobre os casos de coronavírus. A gente ligava para as pessoas que já tinham dado entrada na clínica com suspeita de covid-19 para checar como estavam, se os sintomas tinham melhorado ou não, para ajudar a clínica nesse processo de acompanhar os usuários. ” (Acadêmico 7)

“Junto com a residência de saúde mental, a gente criou um sistema de monitoramento telefônico de usuários de saúde mental [...]. Conseguimos montar um formulário eletrônico e fazer ligações para aqueles que têm um celular. Revezamos isso com os estudantes, com os residentes e os docentes e a gente conseguiu ter uma resposta rápida para fazer um controle dessa população que tem problemas de saúde mental grave e estava isolada na pandemia e, assim, apoiar a atenção básica." (Coordenador de projeto 3) 
“A gente está começando a fazer um telemonitoramento com foco na educação em saúde. Nesse caso é na puericultura, porque a gente não está fazendo acompanhamento de rotina por conta da pandemia. Fazemos ligações com frequência para saber como estão a mãe e a criança e para fazer orientações.” (Preceptor 7).

“Os alunos estão acompanhando grupos de gestantes, diabéticos e hipertensos pelo WhatsApp, evitando que os usuários venham à unidade de forma desnecessária. A enfermeira, preceptora de uma determinada unidade, fez uma lista com os telefones dos usuários com WhatsApp, e o aluno liga e faz um relatório de evolução. Caso não esteja bem, a gente cria um fluxo para essa equipe acionar um agente comunitário para ir até a casa da pessoa." (Tutor 2)

Os grupos tutoriais, em especial os estudantes, também adentraram o mundo das mídias sociais, por meio das quais promovem ações de educação em saúde e engajamento comunitário. Perceberam, nessas mídias, potencial para a introdução de uma nova perspectiva de ensino-aprendizagem, para a disseminação de informações baseadas na ciência e para a manutenção dos vínculos já estabelecidos com a comunidade no primeiro ano de desenvolvimento dos projetos. Nesse âmbito, destacam-se a criação de perfis no Instagram, grupos no WhatsApp e organização de lives e podcasts.

“A gente se organizou para poder fazer peças informativas, com ilustrações, desenhos, para poder fortalecer as informações verdadeiras sobre a pandemia e combater as fake news sobre os remédios, sobre fabricar álcool em gel em casa; e nós compartilhamos em uma rede social, o Instagram. ” (Acadêmico 3)

“Estamos tentando fazer vários movimentos dentro das redes sociais de prevenção contra o coronavírus. Os alunos da nutrição lançam receitas, formas de armazenar comida e isso tudo vai pelo WhatsApp. Tem um outro grupo que fez vários podcasts sobre como é que você limpa os alimentos, a casa, como é que você faz diluição de água sanitária, lavagem das mãos." (Coordenador de projeto 4)

“Já aconteceram algumas lives de preceptores sobre assuntos da atualidade, das experiências que nós estamos vivenciando. Nós também temos alunos do PET-Saúde fazendo vídeos sobre coronavírus, que respondem a dúvidas frequentes da comunidade." (Preceptor 1)

“A gente tem um grupo de WhatsApp que é para tirar dúvidas. A comunidade traz as dúvidas e os preceptores esclarecem. E isso, no início da pandemia, foi muito importante. E, a partir dessas dúvidas, começamos a produzir material para fazer educação popular." (Tutor 5).

As redes sociais também foram palco de divulgação de ações sociais para apoio à comunidade carente, ainda mais necessitada nesses tempos de pandemia.

“A gente organizou uma ação social, uma arrecadação de alimentos para dar suporte para a comunidade que é bem vulnerável. [...] Teve uma listagem, a partir da Clínica da Família, sobre as famílias que mais necessitavam, e aí a gente fez uma campanha pelas mídias sociais para arrecadar esses alimentos. [...] e o pessoal do nosso grupo ajudou a separar esses alimentos e a distribuir as cestas." (Acadêmico 5) 


\section{Discussão}

A rápida disseminação global da covid-19 impôs a todos os países a adoção de medidas urgentes, agressivas e abrangentes de enfrentamento do vírus. Entre as ações de preparação, prontidão e resposta à covid-19 a serem adotadas pelos governos e sistemas de saúde, a Organização Mundial de Saúde sinalizou a importância da comunicação de risco e participação comunitária, a detecção de casos, o rastreamento e gerenciamento de contato, bem como o monitoramento da atividade do vírus nos territórios. Como medidas de saúde pública em nível populacional, recomendou a higienização das mãos e o distanciamento social (World Health Organization, 2020).

No Brasil, o primeiro caso confirmado da doença foi reportado em fevereiro de 2020, no estado de São Paulo. Diante da preocupação com o impacto sanitário, social e econômico na sociedade brasileira, medidas começaram a ser implementadas pelas diversas autoridades políticas, buscando conter a disseminação dos casos e preservar vidas. No âmbito do distanciamento social, que visa a atenuar ou interromper a cadeia de transmissão do vírus, uma das primeiras medidas a serem adotadas pelo Governo Federal, estados e municípios foi a suspensão das atividades acadêmicas presenciais (Silva et al., 2020; Brasil, 2020a), instando as instituições de ensino e educadores a replanejarem o calendário acadêmico e a implementarem atividades de ensino-aprendizagem remotas. Tal fato também impactou nas atividades desenvolvidas pelos grupos tutoriais do PETSaúde/Interprofissionalidade, cuja proposta se baseia no trabalho interativo e colaborativo entre estudantes e profissionais de saúde nos cenários do SUS.

Na conjuntura inicial da pandemia, visando à preservação dos estudantes e constatada a grave interrupção no fornecimento global de equipamentos de proteção individual (EPI) (Cook, 2019), as atividades planejadas nos serviços de saúde foram interrompidas, apesar da discordância de alguns representantes das secretarias de saúde, que acreditavam que os alunos deveriam permanecer nos serviços para apoiar os esforços de enfrentamento da doença, tal como vinha advogando o Governo Federal (Franzoi \& Cauduro, 2020). Este que, por meio do Ministério da Educação, liberou estudantes de medicina, enfermagem, farmácia e fisioterapia a realizarem o estágio curricular obrigatório em unidades de saúde durante a pandemia (Brasil, 2020b); e autorizou antecipação de colação de grau desses estudantes, desde que estivessem no último ano de formação e tivessem cumprido pelo menos $75 \%$ da carga horária prevista para o internato ou estágio supervisionado (Brasil, 2020c). Medidas semelhantes foram adotadas internacionalmente. Na Alemanha, Austrália, Holanda, Jamaica e Dinamarca, por exemplo, incentivou-se a antecipação da formatura e o voluntariado de estudantes de medicina e enfermagem (Rasmussen, Sperling, Poulsen, Emmersen, \& Andersen, 2020; Bourgeault et al., 2020).

Contudo, tais proposições foram rechaçadas pelas entidades profissionais brasileiras e questionadas por estudiosos de outros países em razão da ausência de evidências que apoiassem a adoção de tais medidas, que poderiam significar exposição e transmissão do vírus, desperdício dos já limitados EPI, e risco para a segurança dos estudantes e dos pacientes (Franzoi \& Cauduro, 2020; Hayter \& Jackson, 2020; Menon Klein, Kollars, \& Kleinhenz, 2020).

Fato é que a interrupção das atividades presenciais imprimiu um movimento de replanejamento das ações e a adoção de estratégias e métodos que oportunizassem o desenvolvimento dos projetos. Nesse prisma, a utilização das tecnologias digitais tornou-se fundamental para garantir a dinâmica do ensino e do trabalho remoto. Se, por um lado, perde-se as oportunidades das trocas singulares que acontecem no território de ação, neste caso, nas unidades básicas de saúde e na comunidade; de outro, sempre há um processo de aprendizagem envolvido na produção de novos arranjos, posto que são constituídos a partir de uma ressignificação do contexto analisado coletivamente (Feuerwerker, 2014).

A importância das ferramentas, plataformas e dos ambientes virtuais na aprendizagem já é reconhecida pela literatura. Argumenta-se que os sistemas educativos não acompanham a velocidade e evolução dos saberes, carecendo, portanto, de soluções capazes de ampliar o esforço pedagógico dos formadores. Nessa esteira, advoga-se em favor das tecnologias como 
ferramentas viabilizadoras para o modelo de aprendizagem virtual e a Educação a Distância (EaD) (Lévy, 1999; França, Rabello, \& Magnago, 2019). Para que ocorram e sejam efetivas, no entanto, essas modalidades educacionais imprescindem de planejamento, materiais didáticos apropriados e docentes capacitados (Macebo, 2020). Mais, políticas de acesso a tecnologias e conectividade para docentes, tutores e alunos são requeridas, caso contrário, majora-se o risco do aprofundamento das disparidades educacionais, já que, no Brasil, o acesso à internet e a ferramentas tecnológicas não é usufruto de todos (Carneiro, Rodrigues, França, \& Prata, 2020). Pesquisa nacional revelou que $26 \%$ da população com dez anos ou mais (47 milhões) não acessa à internet; dessas, 45 milhões pertencem às classes econômicas C, D e E (Núcleo de Informação e Coordenação do Ponto $\mathrm{Br}, 2020)$.

No contexto da crise sanitária, a adaptação apressada e improvisada da educação presencial para a EaD, ou melhor para o ensino remoto emergencial (nomenclatura que designa as práticas de ensino virtuais adotadas na pandemia e que não podem ser caracterizadas como EaD, pois esta tem regulamentação própria) (Macebo, 2020) exacerbou as dificuldades enfrentadas por muitos integrantes do PET-Saúde, tal como observado em estudo com docentes de curso de medicina (Kubrusly et al., 2021). Para os alunos de instituições públicas socialmente vulnerabilizadas, além dos desafios relativos à inclusão digital, também foram enunciadas as condições de moradia e de vida, nem sempre compatíveis com a concentração exigida pelos estudos, e o adoecimento de integrantes e/ou de seus familiares acometidos pela covid-19. Resultados semelhantes foram apresentados por estudo envolvendo mais de 500 estudantes de medicina, que também indicou o compartilhamento de equipamentos (computadores e notebooks) com outros membros da família como um limitante para o ensino remoto (Appenzeller et al., 2020).

Não obstante, o uso das tecnologias digitais intensificou os encontros dos grupos tutoriais, agora virtuais, durante os quais se despende mais tempo planejando novas ações a serem desenvolvidas e adaptadas para esse contexto, mas que também funcionam como momentos de acolhimento e de apoio. Sobre isso, a pandemia trouxe muitas mudanças na forma como os indivíduos vivem e se relacionam. Evidências apontam que a crise sanitária elevou o estado de angústia e a prevalência de sofrimento psicológico e de sintomas depressivos entre italianos e estadunidenses, sobretudo nos jovens adultos, pessoas de baixa renda e pessoas expostas a mais estressores, como perda de emprego, problemas financeiros e infecção e/ou morte de alguém próximo por covid-19 (Mazza et al., 2020; Ettman et al., 2020; McGinty, Presskreischer, Han, \& Barry, 2020; Breslau et al., 2021).

No Brasil, os estudos sobre o tema ainda estão em andamento; mas, no Rio Grande do Sul, constatou-se que ter renda diminuída durante a pandemia, ser do grupo de risco e estar mais exposto a informações negativas sobre a crise sanitária são fatores que podem provocar maior prejuízo na saúde mental (Duarte, Santo, Lima, Giordani, \& Trentini, 2020). Entre estudantes de medicina, a prevalência com indícios de sofrimento psíquico foi de $62,8 \%$; e a proporção dos que apresentaram ideação suicida foi de $9,1 \%$. Entre os fatores de risco correlacionados estão a má adaptação ao ensino a distância, dificuldade de concentração, morar com alguém que precisa trabalhar fora de casa, ser incapaz de manter hábitos saudáveis e ter medo de ser infectado pelo vírus (Maia \& Dias, 2020).

É preciso ressaltar que um dos componentes dos grupos tutoriais do PET-Saúde, o preceptor, precisa não apenas enfrentar os desafios cotidianos do trabalho, que incluem infraestrutura e condições laborais precárias e sobrecarga de trabalho, mas dispor de mais energia e capacidade de resiliência para experienciar as adversidades da pandemia. Além de estarem mais suscetíveis ao adoecimento e morte por covid-19, os profissionais de saúde também estão mais propensos ao desenvolvimento de sofrimento mental (Lai et al., 2019; Bohlken, Schömig, Lemke, Pumberger, \& Riedel-Heller, 2020).

No Brasil, essa situação se reflete no cenário epidemiológico da pandemia entre os trabalhadores da saúde. Nessa população, até o dia 29 de março de 2021 foram notificados mais de 240 mil casos de síndrome gripal suspeitos de covid-19 no 
e-SUS Notifica. Destes, 28,7\% foram confirmados. O número de óbitos disponibilizado pelo Ministério da Saúde não reflete o total dos acometidos pela doença no país, mas se sabe que pelo menos 220 profissionais morreram em decorrência de síndrome respiratória aguda grave causada pelo Sars-CoV-2 (Brasil, 2021). O Conselho Federal de Enfermagem (2021) e o Conselho Federal de Medicina (2021) registraram até o momento, 14 de abril de 2021, 763 e 727 óbitos de profissionais de enfermagem e de médicos, respectivamente.

Sobre a saúde mental, dados preliminares do estudo Condições de Trabalho dos Profissionais de Saúde no Contexto da Covid-19, realizado pelo Fundação Oswaldo Cruz, apontam que, dos mais de 15 mil entrevistados, 43,2\% não se sentem protegidos no trabalho de enfrentamento da doença, $18 \%$ sentem medo generalizado de se contaminar no trabalho e $14 \%$ estão no limite da exaustão. As alterações no cotidiano mais relatadas foram perturbação do sono, irritabilidade, choro frequente, incapacidade de relaxar e estresse. Pensamentos negativos, incluindo ideação suicida, foram reportados por 8,3\% (Leonel, 2021).

Fato é que a incerteza, o medo, as rotinas diárias alteradas e o isolamento social afetaram drasticamente a vida das pessoas, sendo recomendável que elas contêm umas com as outras para conexões e estratégias de enfrentamento para aliviar o peso da crise em sua saúde mental (Li et al., 2021). Muitas pesquisas demonstraram o efeito protetor do suporte social percebido na saúde mental em situações estressantes (Kawachi \& Berkman, 2001; Khan \& Husain, 2010; Eisman, Stoddard, Heinze, Caldwell, \& Zimmerman, 2015). Nessa perspectiva, a conformação de uma rede de apoio no contexto do PET-Saúde pode ser considerada estratégia humanizadora e potente para o arrefecimento da solidão e da angústia.

Os resultados de nosso estudo também mostraram que a pandemia instou os grupos tutoriais a apoiar as secretarias de saúde no enfrentamento da pandemia. A ação de apoio predominante foi o monitoramento de usuários por meio de ligações telefônicas e aplicativos de mensagem. Esse telemonitoramento não se restringiu aos casos de coronavírus; também incluiu o seguimento de gestantes, crianças, usuários de saúde mental e pessoas com doenças crônicas não transmissíveis. A participação de estudantes da saúde em atividades de monitoramento remoto durante a pandemia também foi reportada por outras pesquisas (Menezes, Freitas, Pedreira, \& Amaral, 2020; Silveira et al., 2021).

$\mathrm{Na}$ impossibilidade de atuação presencial nos campos de prática, a realização do telemonitoramento possibilitou aos alunos a vivência de ações de cuidado e o desenvolvimento de competências com correspondência na atenção básica, a exemplo da comunicação, educação em saúde e vigilância em saúde. Mais, as ações também permitiram a manutenção do vínculo com a comunidade, evitou que os usuários ficassem desassistidos e que buscassem as unidades básicas desnecessariamente. Outros benefícios do monitoramento remoto citados pela literatura incluem a otimização das agendas dos profissionais de saúde para o atendimento presencial (Rodrigues et al., 2020), identificação precoce de problemas de saúde, redução dos gastos, otimização da capacidade de atenção e, no caso da covid-19, minimização do risco de transmissão viral (Silven et al., 2020; Boggan et al., 2020).

Os grupos tutoriais também investiram na educação em saúde por meio das mídias sociais, sobretudo Instagram, WhatsApp e Youtube. Por serem gratuitas e removerem as barreiras físicas que tradicionalmente impedem o acesso a recursos e suporte de saúde, essas redes sociais facilitam a interatividade, o consumo e o compartilhamento de informações de saúde (Curran et al., 2017; Markham, Gentile, \& Graham, 2017; Ladaga Andrade, Sartori, \& Yamaguchi, 2018). Por outro lado, as mídias digitais também contribuem para o fenômeno da infodemia, termo atribuído ao efeito negativo do crescente volume de informações sobre um determinado tema, nem sempre verdadeiras, que podem se multiplicar e disseminar em um curto período, e levar instituições e pessoas a tomar decisões contraproducentes para os interesses coletivos (Zarocostas, 2020).

No cenário pandêmico brasileiro, o negacionismo científico - que tem como seu principal expoente o Presidente da República - intensificou a propagação maciça de notícias falsas e rumores sobre a covid-19, vacinas e medidas de prevenção, 
outro desafio a ser enfrentado pelos cientistas e profissionais de saúde nesta Era da Informação (Dyer, 2020; Galhardi, Freire, Minayo, \& Fagundes., 2020; Ventura \& Reis, 2021). Nessa conjuntura, os petianos se valeram das mídias sociais para disseminar informações baseadas na ciência. Lives, podcasts e posts foram produzidos interprofissionalmente por estudantes de diferentes cursos, com o apoio dos tutores e preceptores, a fim de conscientizar a população sobre a importância da vacinação, higienização das mãos, distanciamento social, uso de máscaras e ineficácia do chamado "tratamento precoce" - financiado e difundido pelo Governo Federal (Francisco \& Michael, 2020).

Acredita-se que o uso das mídias sociais como plataformas de educação popular pode aproximar a comunidade acadêmica da população e contribuir para o fomento de atitudes comunitárias benéficas à saúde coletiva. Isto pois, no cenário pandêmico, a educação em saúde tem o potencial de promover a consciência crítica da sociedade a respeito da doença, estimulando a adoção de medidas preventivas individuais que ainda são as principais armas contra a disseminação do vírus. Pontua-se, no entanto, que essas ações podem não alcançar os usuários digitalmente excluídos, o que torna imprescindível o emprego de métodos diversificados para a disseminação de informações.

\section{Considerações Finais}

Consoantes às orientações científicas internacionais de distanciamento social e certos da importância de manter-se a integração ensino-serviço-comunidade, os integrantes do PET-Saúde/Interprofissionalidade precisaram replanejar suas atividades para o ambiente virtual. Para tanto, se alicerçaram no uso das tecnologias de comunicação digitais para a promoção de encontros semanais, nos quais o acolhimento e o apoio mútuo têm abrandado a sensação de solidão.

Até o momento da coleta de dados, as estratégias de monitoramento remoto de usuários e a educação em saúde nas mídias sociais eram as ações de enfrentamento da covid-19 mais desenvolvidas no âmbito dos projetos PET-Saúde do estado do Rio de Janeiro. Os diferentes movimentos de adaptação dos grupos tutoriais ao contexto pandêmico permitiram a continuidade segura dos projetos, apoio ao combate da doença, manutenção do vínculo com a comunidade e promoveu o despertar da criatividade e da empatia.

Os movimentos empreendidos pelos grupos tutoriais reafirmam a integração ensino-serviço-comunidade como fio condutor dos processos de reorientação da formação. Mais, as ações desempenhadas, planejadas e estruturadas coletivamente, respeitando-se as limitações e vulnerabilidades dos estudantes, preceptores e tutores, demonstram que é possível apoiar o enfrentamento da pandemia de covid-19, e ensinar e aprender a partir da realidade cotidiana dos serviços de saúde, mesmo não estando lá, na linha de frente.

Pesquisas como estas vêm demonstrando a potência do PET-Saúde na reordenação da formação de profissionais para o SUS, no fortalecimento do diálogo entre o ensino e o serviço e na transformação das práticas de saúde; por outro lado, inexistem evidências sobre os impactos do programa na atuação profissional dos egressos que dele participaram e nos indicadores de saúde. Recomenda-se, nesse sentido, que esta lacuna seja preenchida a partir de pesquisas de monitoramento e avaliação contínuas, que sejam abrangentes e robustas, e desenvolvidas em parceria com os serviços de saúde.

\section{Referências}

Almeida, R. G. S., Teston, E. F., \& Medeiros, A. A. (2019). A interface entre o PET-Saúde/Interprofissionalidade e a Política Nacional de Educação Permanente em Saúde. Saúde em Debate, 43(spe1), 97-105. 10.1590/0103-11042019s108

Appenzeller, S., Menezes, F. H., Santos, G. G., Padilha, R. F., Graça, H. S., \& Bragança, J. F. (2020). Novos tempos, novos desafios: estratégias para equidade de acesso ao ensino remoto emergencial. Revista Brasileira de Educação Médica, 44(supl. 1), e155. 10.1590/1981-5271v44.supl.1-20200420

Bardin, L. (2011). Análise de conteúdo. São Paulo: Edições 70. 
Barr, H. (1998). Competent to collaborate: towards a competency-based model for interprofessional education. Journal of Interprofessional Care, 12(2), 181187. $10.3109 / 13561829809014104$

Boggan, J. C., Shoup, J. P., Whited, J. D., Voorhees, E. V., Gordon, A. M., Rushton, S., ...Gierisch, J. M. (2020) Effectiveness of acute care remote triage systems: a systematic review. Journal of General International Medicine, 35(7), 2136-2145. 10.1007/s11606-019-05585-4

Bohlken, J., Schömig, F., Lemke, M. R., Pumberger, M., \& Riedel-Heller, S. G. (2020). COVID-19-Pandemie: Belastungen des medizinischen Personals [COVID-19 Pandemic: Stress Experience of Healthcare Workers - A Short Current Review]. Psychiatrische Praxis, 47(4), 190-197. 10.1055/a-1159-5551

Bourgeault, I. L., Maier, C. B., Dieleman, M, Ball, J., MacKenzie, A., Nancarrow, S., ... Sidat, M. (2020). The COVID-19 pandemic presents an opportunity to develop more sustainable health workforces. Human Resources for Health, London, 18, 83. 10.1186/s12960-020-00529-0

Brasil (2020a). Ministério da Educação. Portaria nº 343, de 17 de março de 2020. Diário Oficial da República Federativa do Brasil. Seção 1, 39.

Brasil (2020b). Ministério da Educação. Portaria n 356, de 20 de março de 2020. Diário Oficial da República Federativa do Brasil, Seção 1, 1.

Brasil (2020c) Ministério da Educação. Portaria no 383 de 09 de abril de 2020. Diário Oficial da República Federativa do Brasil, Seção 1, 24

Brasil (2021). Ministério da Saúde. Secretaria de Vigilância em Saúde. Boletim Epidemiológico Especial: doença pelo coronavírus - COVID-19, (57). Brasília: Ministério da Saúde. 〈https://www.gov.br/saude/pt-br/media/pdf/2021/abril/08/boletim_epidemiologico_covid_57.pdf〉.

Breslau, J., Finucane, M. L., Locker, A. R., Baird, M. D., Roth, E. A., \& Collins, R. L. (2021). A longitudinal study of psychological distress in the United States before and during the COVID-19 pandemic. Preventive Medicine, 143, 106362. 10.1016/j.ypmed.2020.106362

Carneiro, L. A., Rodrigues, W., França, G., Prata, D. N. (2020). Uso de tecnologias no ensino superior público brasileiro em tempos de pandemia COVID-19. Research, Society and Development, 9(8), e267985485. 10.33448/rsd-v9i8.5485

Conselho Federal de Enfermagem (2021). Observatório da Enfermagem. <http://observatoriodaenfermagem.cofen.gov.br/>.

Conselho Federal de Medicina (2021). Memorial aos médicos que se foram durante o combate à COVID-19. <https://memorial.cfm.org.br/>.

Conselho Nacional de Secretários de Saúde. Painel Conass Covid-19. <https://www.conass.org.br/painelconasscovid19/>.

Cook, T. M. (2020). Personal protective equipment during the coronavirus disease (COVID) 2019 pandemic - a narrative review. Anaesthesia, 75(7), 920-927. 10.1111/anae. 15071

Curran, V., Matthews, L., Fleet, L., Simmons, K., Gustafson, D. L., \& Wetsch, L. (2017). A Review of Digital, Social, and Mobile Technologies in Health Professional Education. The Journal of Continuing Education in the Health Professions, 37(3), 195-206. 10.1097/CEH.0000000000000168

Duarte, M. Q., Santo, M. A. S., Lima, C. P., Giordani, J. P., \& Trentini, C. M. (2020). Covid-19 and the impacts on mental health: a sample from Rio Grande do Sul, Brazil. Ciência \& Saúde Coletiva, 25(9), 3401-3411. 10.1590/1413-81232020259.16472020

Dyer, O. (2020). Covid-19: Bolsonaro under fire as Brazil hides figures. The BMJ, 369, m2296. 10.1136/bmj.m2296

Eisman, A. B., Stoddard, S. A., Heinze, J., Caldwell, C. H., \& Zimmerman, M. A. (2015). Depressive symptoms, social support, and violence exposure among urban youth: A longitudinal study of resilience. Developmental psychology, 51(9), 1307-1316. 10.1037/a0039501

Ettman, C. K., Abdalla, S. M., Cohen, G. H., Sampson, L., Vivier, P. M., \& Galea, S. (2020). Prevalence of Depression Symptoms in US Adults Before and During the COVID-19 Pandemic. JAMA Network Open, 3(9), e2019686. 10.1001/jamanetworkopen.2020.19686

Feuerwerker, L. C. M. (2014). Micropolítica e saúde: produção do cuidado, gestão e formação. Porto Alegre: Rede UNIDA.

França, T., Magnago, C., Santos, M. R., Belisário, S. A., \& Silva, C. B. G. (2018). PET-Saúde/GraduaSUS: retrospectiva, diferenciais e panorama de distribuição dos projetos. Saúde em Debate, 42(spe2), 286-301. 10.1590/0103-11042018s220

França, T., Rabello, E. T., \& Magnago, C. (2019). Digital media and platforms in the Permanent Health Education field: debates and proposals. Saúde em Debate, 43(spe1), 106-115. https://doi.org/10.1590/0103-11042019s109

Francisco O., \& Michael, O. (2020). Governing COVID-19 without government in Brazil: Ignorance, neoliberal authoritarianism, and the collapse of public health leadership. Global Public Health, 15(9):1257-1277. 10.1080/17441692.2020.1795223

Franzoi, M. A. H., \& Cauduro, F. L. F. (2020). Participation of nursing students in the COVID-19 pandemic. Cogitare Enfermagem, 25, e73491. $10.5380 /$ ce.v25i0.73491

Galhardi, C. P., Freire, N. P., Minayo, M. C. S., \& Fagundes, M. C. M. (2020). Fact or Fake? An analysis of disinformation regarding the Covid-19 pandemic in Brazil. Ciência \& Saúde Coletiva, 25(suppl 2), 4201-4210. 10.1590/1413-812320202510.2.28922020

Garcia, L. P., \& Duarte, E. (2020). Intervenções não farmacológicas para o enfrentamento à epidemia da Covid-19 no Brasil. Epidemiologia e Serviços de Saúde, 29, e2020222. 10.5123/s1679-49742020000200009

Hayter, M., \& Jackson, D. (2020). Pre-registration undergraduate nurses and the COVID-19 pandemic: Students or workers? Journal of Clinical Nursing, 29(17/18), 3115-3116. 10.1111/jocn.15317

Kawachi, I,.L., \& Berkman, L. F. (2001). Social ties and mental health. Journal of Urban Health, 78, 458-467. 10.1093/jurban/78.3.458 
Khan, A., \& Husain, A. (2010). Social support as a moderator of positive psychological strengths and subjective well-being. Psychological Reports, 106, 3438. 10.2466/PR0.106.2.534-538

Kubrusly, M., Coelho, R. A., Augusto, K. L., Peixoto Junior, A. A., Santos, D. C. O., \& Oliveira, C. M. C. (2021). Percepção docente sobre a aprendizagem baseada em problemas no ensino remoto durante a pandemia de COVID-19. Research, Society and Development, 10(5), e53510515280. 10.33448/rsdv10i5.15280

Ladaga, F. M., Andrade, G., Sartori, A. C., \& Yamaguchi, M. (2018). WhatsApp, uma ferramenta emergente para a promoção da saúde. Enciclopédia Biosfera, 15(28), 1370-1384.

Lai, J., Ma, S., Wang, Y., Cai, Z., Hu, J., Wei, N., ... Hu, S. (2020). Factors Associated with Mental Health Outcomes Among Health Care Workers Exposed to Coronavirus Disease 2019. JAMA Network Open, 3(3), e203976. 10.1001/jamanetworkopen.2020.3976

Leonel, F. (2021). Pesquisa analisa o impacto da pandemia entre profissionais de saúde. Fundação Oswaldo Cruz, 22 mar. 2021. <https://portal.fiocruz.br/noticia/pesquisa-analisa-o-impacto-da-pandemia-entre-profissionais-de-saude>. Recuperado em: 20 abr. 2021.

Lévy, P. (1999). Cibercultura. São Paulo: Editora 34.

Li, F., Luo, S., Mu, W., Li, Y., Ye, L., Zheng, X., ... Chen, X. (2021). Effects of sources of social support and resilience on the mental health of different age groups during the COVID-19 pandemic. BMC Psychiatry, 21(1), 16. 10.1186/s12888-020-03012-1

Macebo, D. (2020). Trabalho remoto na Educação Superior brasileira: efeitos e possibilidades no contexto da pandemia. Revista USP, 127, 105-116. $10.11606 /$ issn.2316-9036.i127p105-116

Maia, B. R., \& Dias, P. C. (2020). Ansiedade, depressão e estresse em estudantes universitários: o impacto da COVID-19. Estudos de Psicologia, 37 , e200067. $10.1590 / 1982-0275202037 \mathrm{e} 200067$

Markham, M. J., Gentile, D., \& Graham, D. L. (2017). Social Media for Networking, Professional Development, and Patient Engagement. American Society of Clinical Oncology Educational Book, 37, 782-787. 10.14694/EDBK_180077

Mazza, C., Ricci, E., Biondi, S., Colasanti, M., Ferracuti, S., Napoli, C., \& Roma, P. (2020). A Nationwide Survey of Psychological Distress among Italian People during the COVID-19 Pandemic: Immediate Psychological Responses and Associated Factors International Journal of Environmental Research and Public Health, 17(9), 3165. 10.3390/ijerph17093165

McGinty, E. E., Presskreischer, R., Han, H., \& Barry, C. L. (2020). Psychological distress and loneliness reported by US adults in 2018 and April 2020. JAMA Network Open, 324(1), 93-94. 10.1001/jama.2020.9740

Menezes, T., Freitas, A. V. S., Pedreira, L. C., \& Amaral, J. B. (2020). Telemonitoring of Brazilian Nursing homes before Coronavirus and COVID-19 Infections. Revista Brasileira de Enfermagem, 73(suppl 2), e20200350. 10.1590/0034-7167-2020-0350

Menon, A., Klein, E. J., Kollars, K., \& Kleinhenz, A. L. W. (2020). Medical Students Are Not Essential Workers: Examining Institutional Responsibility During the COVID-19 Pandemic. Academic Medicine, 95(8):1149-1151. 10.1097/ACM.0000000000003478

Núcleo de Informação e Coordenação do Ponto $\mathrm{Br}$ (2020). Pesquisa sobre o uso das tecnologias de informação e comunicação nos domicílios brasileiros: TIC Domicílios 2019. São Paulo: Comitê Gestor no <https://www.cetic.br/media/docs/publicacoes/2/20201123121817/tic_dom_2019_livro_eletronico.pdf>.

Rasmussen, S., Sperling, P., Poulsen, M. S., Emmersen, J., \& Andersen, S. (2020). Medical students for health-care staff shortages during the COVID-19 pandemic. Lancet, 395(10234), e79-e80. 10.1016/S0140-6736(20)30923-5

Reeves, S. (2016). Why we need interprofessional education to improve the delivery of safe and effective care. Interface - Comunicação, Saúde, Educação, 20(56), 185-197. 10.1590/1807-57622014.0092

Rodrigues, A. P., Felipe, C. R., Lima, D. B., Costa, L. R. O., Fernandes, P. F., Silva, R. P. P., ... Lazarini, W. S. (2020). Telemonitoramento como estratégia de cuidado longitudinal a grupos prioritários em tempos da COVID-19: uma experiência na atenção primária à saúde do município de Vitória-ES. APS em Revista, 2(2), 189-196. 10.14295/aps.v2i2.100

Silva, L. L. S., Lima, A. F. R., Polli, D. A., Razia, P. F. S., Pavão, L. F. A., Cavalcanti, M. A. F. H., \& Toscano, C. M. (2020). Social distancing measures in the fight against COVID-19 in Brazil: description and epidemiological analysis by state. Cadernos de Saúde Pública, 36(9), e00185020. 10.1590/0102$311 \times 00185020$

Silveira, R. P., Costa, J. M., França, S. S., Pereira, R. C. R., Lomonaco, L. A., \& Leal Junior, O. S. (2021). Projeto de ensino como apoio ao telemonitoramento dos casos de Covid-19. Revista Brasileira de Educação Médica, 45(1), e050. 10.1590/1981-5271v45.1-20200319

Silven, A. V., Petrus, A., Villalobos-Quesada, M., Dirikgil, E., Oerlemans, C. R., Landstra, C. P., ... Teng, Y. (2020). Telemonitoring for Patients with COVID-19: Recommendations for Design and Implementation. Journal of Medical Internet Research, 22(9), e20953. 10.2196/20953

Ventura, D. F. L., \& Reis, R. (2021). A linha do tempo da estratégia federal de disseminação da covid-19. Boletim Direitos na Pandemia: mapeamento e análise das normas jurídicas de resposta à covid-19 no Brasil, (10), p. 6-31.: <https://static.poder360.com.br/2021/01/boletim-direitos-na-pandemia.pdf>.

World Health Organization (2020). Critical preparedness, readiness and response actions for COVID-19: interim guidance. Geneva: World Health Organization.

Zarocostas, J. (2020). How to fight an infodemic. Lancet, 395(10225), 676. 10.1016/S0140-6736(20)30461-X 